\title{
Active Noise Control for Three-Dimensional Sound Field Using Fast Converging Algorithms*
}

\author{
Shinya KIJIMOTO**, Yuichi SASAKI ${ }^{* * *}$, Yoichi KANEMITSU**, \\ Koichi MATSUDA** and Takashi YOSHIDA ${ }^{\dagger}$ \\ ** Department of Intelligent Machinery and Systems, Kyushu University \\ 744 Motooka, Nishi-ku, Fukuoka, 819-0395, Japan \\ E-mail: kiji@mech.kyushu-u.ac.jp \\ *** Yamaha Motor CO., LTD., Japan \\ 2500 Shingai, Iwata-shi, Shizuoka, 438-8501, Japan \\ † Department of Electrical and Electronic Systems Engineering, Kyushu University \\ 744 Motooka, Nishi-ku, Fukuoka, 819-0395, Japan
}

\begin{abstract}
Active control in three-dimensional sound field against incoming noise from external noise source is considered. Convergence characteristics are investigated with not only conventionally wide-used Filtered-x LMS algorithm, but also fast transversal filter (FTF) algorithm and frequency domain adaptive (FDA) algorithm. The advantages and disadvantages of each algorithm is examined through simulations and experiments using 1/5 scale model of a general room. Control results are studied also with both fixed noise source and moving noise source, and the difference in the control performance of each algorithm is compared and considered. As a result of simulations and experiments, both FTF and FDA show better control performance than LMS algorithm against the fixed noise source. Also while noise source is moving, FTF shows better results than LMS does.
\end{abstract}

Key words : Active Noise Control, Filtered-x LMS Algorithm, Fast Transversal Filter Algorithm, Frequency Domain Adaptive Algorithm

\section{Introduction}

Influence of the noise causes many problems for a human being. There are two approaches, passive and active, for noise control. The active noise control is effective against the low frequency noise. In this paper, the active noise control technique in three-dimensional sound field is investigated. The authors carried out simulations and experiments about active noise control against incoming noise from an external noise source ${ }^{(1),(2)}$.

As the external noise source, a moving source like cars or airplanes is more general than a fixed source. When active acoustic control is applied against such moving noise sources, it is important that adaptation speed of adaptive algorithm is high.

A purpose of this paper is an active noise control in a three-dimensional sound field against incident noise. In the active noise control, Filtered-x LMS algorithm ${ }^{(3)}$ is generally used by its stability and brevity. However, Filtered-x LMS algorithm has a defect of slow convergence speed. On the other hand, it is known that there are some algorithms which have high-speed convergence characteristics.

In this paper, convergence characteristics of Fast Transversal Filter (FTF) $\operatorname{algorithm}^{(4),(5)}$ and Frequency Domain Adaptive (FDA) algorithm ${ }^{(6)}$ are compared with conventional Filtered$x$ LMS algorithm through both simulations and experiments. Until now, the authors confirmed applicability to active noise control of FTF algorithm ${ }^{(7)}$. Figs. 1 and 2 show schematic diagrams of sound field considered in this paper. Fig. 1 shows the sound field with the fixed noise source, and Fig. 2 shows it with the moving noise source. A control system consists of a reference microphone, a control sound source, and an error microphone. Each control

*Received 27 Dec., 2006 (No. T-04-0953) Japanese Original: Trans. Jpn. Soc. Mech. Eng., Vol.71, No.707, C (2005), pp.2141-2146 (Received 27 Aug., 2004) [DOI: 10.1299/jee.2.257] 
element is placed as shown in Figs. 1 and 2.

\section{Adaptive Algorithms}

There are two types of adaptive algorithms. One is a gradient method, and the other is a least squares method. LMS belongs to the former type, and has characteristics of less computational complexity and high stability, but on the other hand, it has slow convergence speed. FTF and FDA belong to the latter type, the least squares method, so their computational complexity increases, but convergence speed is fast.

\subsection{Filtered-x LMS algorithm}

A block diagram of filtered-x LMS algorithm is shown in Fig. 3. In the figure, $W$ is an acoustic characteristic from the reference microphone to the control point (the position of the

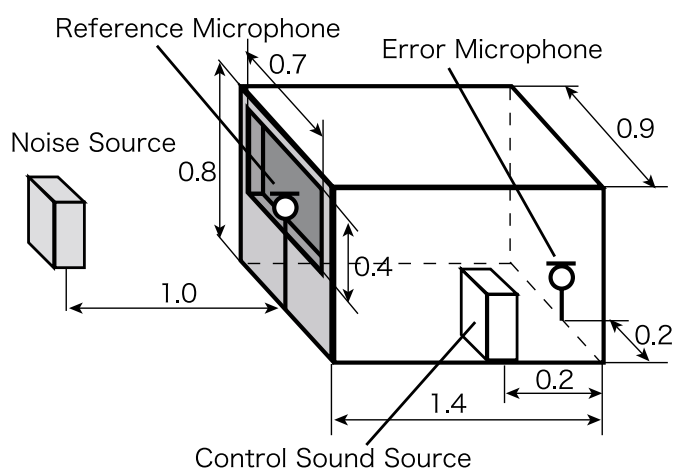

Fig. 1 3D sound field with fixed noise source

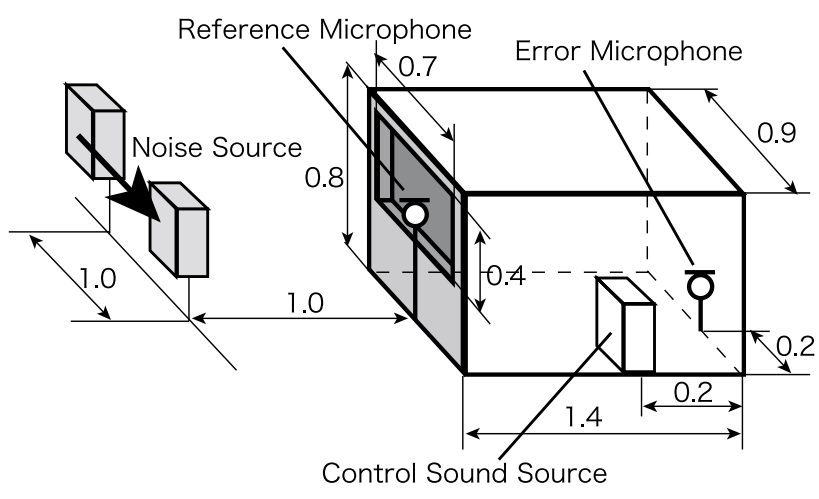

Fig. 2 3D sound field with moving noise source

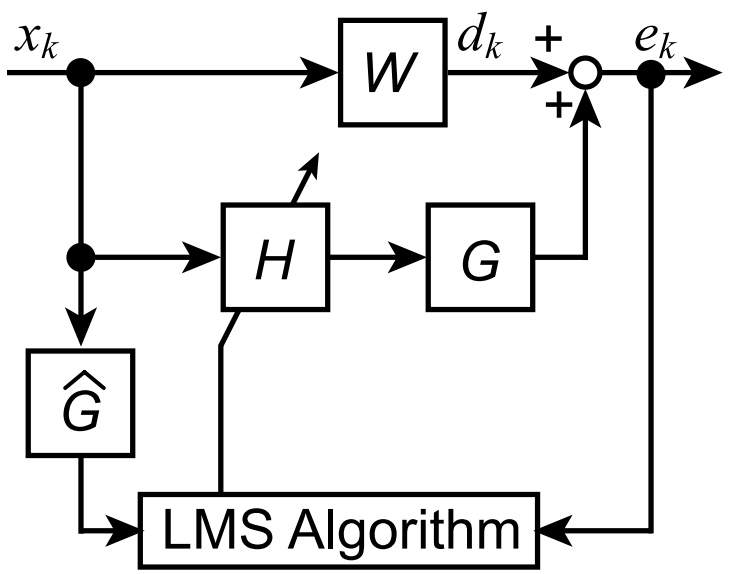

Fig. 3 Block diagram of filtered-x LMS 
error microphone), $G$ is an acoustic characteristic from the control sound source to the control point, $\hat{G}$ is an estimated characteristic of $G$, and $H$ is a transfer characteristic of the controller. The update equation of filtered-x LMS algorithm is given by

$$
\boldsymbol{h}_{k+1}=\boldsymbol{h}_{k}-2 \mu e_{k} \widehat{\boldsymbol{g}} * \boldsymbol{x}_{k},
$$

where $\boldsymbol{h}_{k}$ is the vector of controller filter coefficients at the $k$ th sample, $\boldsymbol{\mu}$ is the stepsize parameter, $e_{k}$ is the error signal, $\widehat{\boldsymbol{g}}$ is the coefficients vector of FIR filter $\hat{\boldsymbol{G}}$, and $\boldsymbol{x}_{k}$ is the vector of the input signals. In the eq. (1), “*” denotes the convolution.

\subsection{FTF algorithm}

A block diagram of FTF algorithm is shown in Fig. 4. In the FTF algorithm, Input/output signal of the system is necessary, so the filtered control output by the estimated characteristic $\hat{G}$ is subtracted from the error signal for obtaining estimated output signal of the system as shown in Fig. 4.

\subsection{FDA algorithm}

A block diagram of FDA algorithm is shown in Fig. 5. FDA algorithm transforms each signal into frequency domain and calculates transfer characteristic of the controller by using $H_{1}$ estimation $^{(8)}$ as shown in Fig. 5. Same as FTF (Fig. 4), input/output signal of the system is necessary in the FDA algorithm.

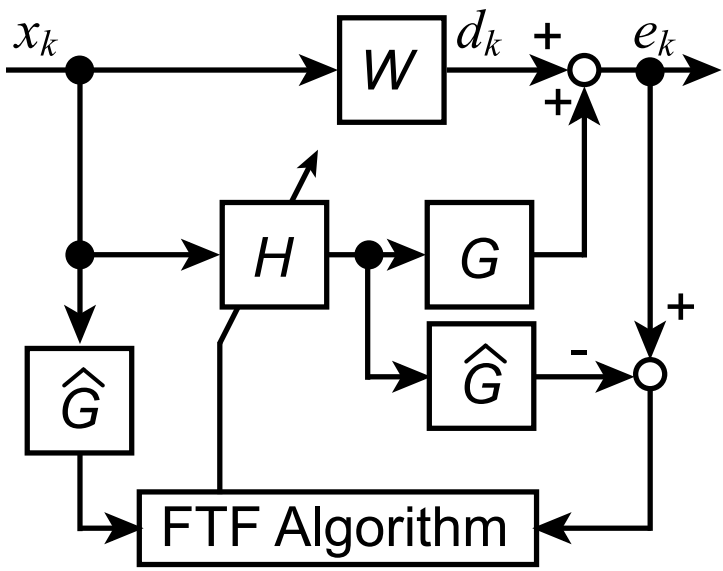

Fig. 4 Block diagram of FTF

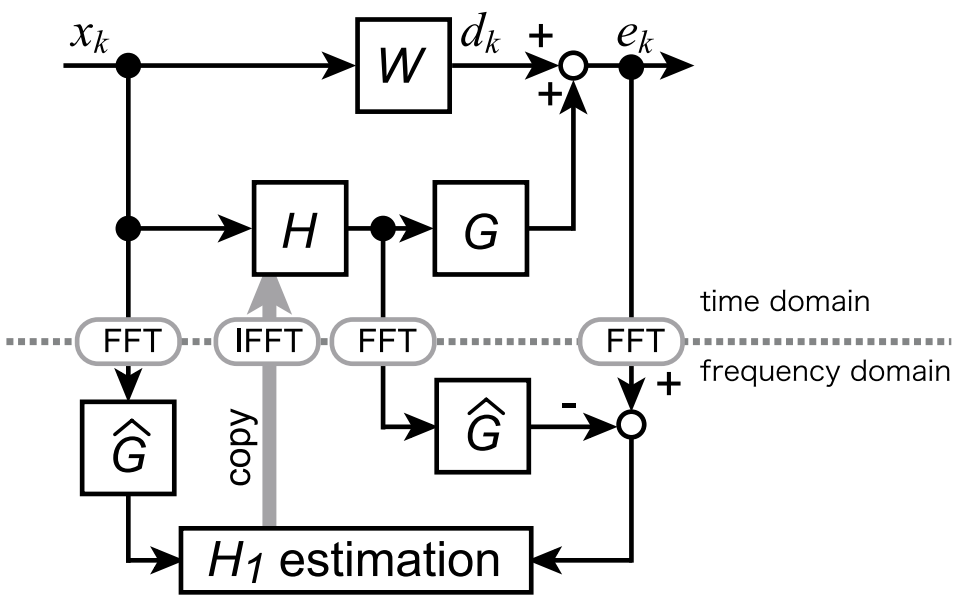

Fig. 5 Block diagram of FDA 


\section{Improvement of Adaptive Algorithms}

In this paper, FTF and FDA are improved as follows.

\subsection{FTF algorithm}

3.1.1. Measures against initial instability In the early stage of the FTF algorithm, it is necessary to solve the simultaneous equation given by

$$
\left[\begin{array}{c}
d_{0} \\
d_{1} \\
\vdots \\
d_{p-1} \\
d_{p}
\end{array}\right]=\left[\begin{array}{ccccc}
x_{0} & & & & \\
x_{1} & \ddots & & \mathbf{0} & \\
\vdots & & \ddots & & \\
x_{p-1} & & & \ddots & \\
x_{p} & x_{p-1} & \ldots & x_{1} & x_{0}
\end{array}\right]\left[\begin{array}{c}
h_{0} \\
h_{1} \\
\vdots \\
h_{p-1} \\
h_{p}
\end{array}\right]
$$

where $d_{k}, x_{k}, h_{k}$ are output signal, input signal, and filter coefficients of the controller at $k$ th sample respectively, and $p$ is a filter length of the controller. The input signal matrix in this equation is the Toeplitz matrix. If diagonal element $x_{0}$ is not large enough, the matrix will be ill-conditioned, and the filter coefficients will diverge. To avoid this instability, we forcibly let the diagonal element $x_{0}$ be a large value. Although the filter coefficient $h$ obtained through this approach will be smaller than the optimum coefficient, it will converge to the optimum value by the subsequent adaptation process.

3.1.2. Measures against quantization error In FTF algorithm, it is known that filter coefficient can diverge during adaptation process by influence of a quantization error. To avoid this divergence caused by the quantization error, updating will be suspended at the suitable point during adaptation. In this paper, an estimation error (see Eq. (4)) which is an index to convergence is used as a criterion of suspending update instead of the rescue variable ${ }^{(4)}$ or the error feedback ${ }^{(4)}$. Fig. 6a shows a simulation result not using estimation error and Fig. $6 \mathrm{~b}$ shows a result using estimation error. From these figures, it is shown that using the estimation error as a criterion is effective for suppressing divergence. However, adaptability is lost when update is completely stopped. Therefore, batch-type adaptation process is proposed in this paper. In this process, filter coefficients of the controller are not updated at every sampling, but are copied from the identification section after each convergence of adaptive identification.

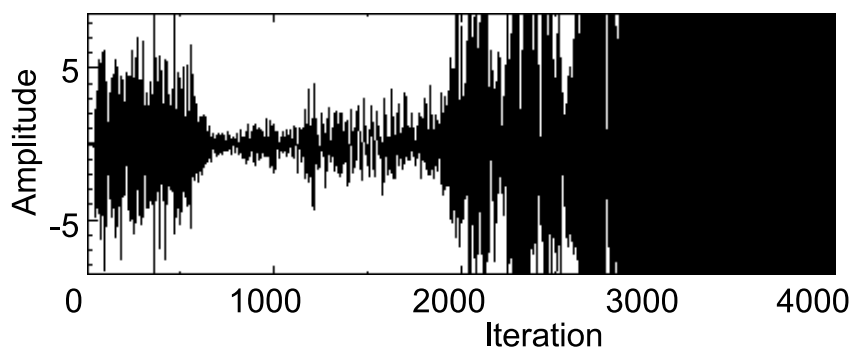

a. without using estimation error

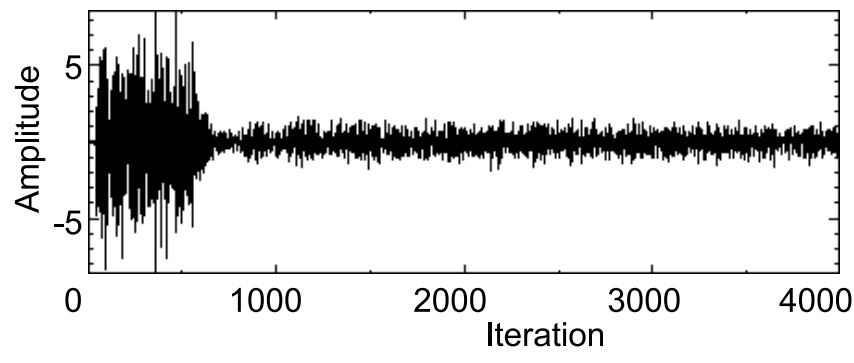

b. using estimation error

Fig.6 Quantization error 
By this process, convergence speed becomes slower than ordinary FTF, but the control system will keep stability during controlling.

\subsection{FDA algorithm}

In FDA algorithm, the averaging is carried out in the $H_{1}$ estimation. In this averaging process, it is assumed that the system is time-invariant, so the moving noise sources cannot be considered. Therefore, a forgetting factor is newly introduced into this averaging process. The new averaging equation is given by

$$
\overline{X_{k}}=\frac{\left(\lambda^{k-1} X_{1}+\cdots+\lambda X_{k-1}+X_{k}\right)}{1+\lambda+\cdots+\lambda^{k-1}}
$$

where $X_{k}$ is cross-spectral density function between input and output signals or power spectral density of input signals at the $k$ th sample, and $\lambda$ is the forgetting factor and is set the value of 0.8 in this paper. By this improvement, FDA algorithm will be able to deal with a change of an acoustic characteristic even after converging.

\section{Simulations}

Conditions of simulations in this paper are as follows.

( 1 ) Acoustic characteristics $W$ and $G$ are identified as FIR filters through inverse Fourier transformation from measured value of the sound field characteristics in the $1 / 5$ scale model shown in Figs. 1 or 2.

(2) Filter lengths of FIR filters are 512.

( 3 ) White noise is used in the simulation.

(4) A feedback path, from the control source to the reference microphone, is not considered in the simulation.

( 5 ) Following estimation error is used as an index to evaluating a control effect.

$$
E E=10 \log _{10} \frac{\sum e^{2}}{\sum d^{2}}
$$

\subsection{Fixed noise source}

Figure 7 shows results of simulations about fixed noise source as shown in Fig. 1. A horizontal axis means the iteration number of update from a beginning of control, and a vertical axis means the estimation error expressed in Eq. (4). From Fig. 7, it is obvious that the convergence speeds of FTF and FDA are faster than it of LMS.

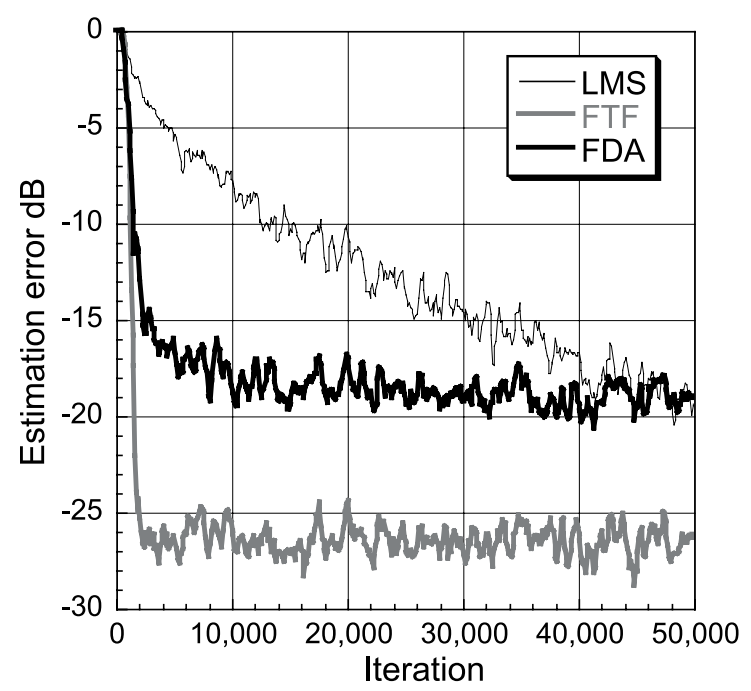

Fig. 7 Simulation: fixed noise source 


\subsection{Moving noise source}

Figures 8 and 9 show results of simulations about moving noise source as shown in Fig. 2. Figures 8 and 9 show the results when the noise source is assumed to have a speed of $0.1 \mathrm{~m} / \mathrm{s}$ and $0.25 \mathrm{~m} / \mathrm{s}$ respectively. The dark part of each figures shows that the noise source is moving during that part. Comparing with the results of the fixed noise source (see Fig. 7), control effects for moving noise source become worse, but, we can see from Figs. 8 and 9 that both FTF and FDA algorithms are stable during control. We can also find out that although the result of LMS in higher speed becomes slightly unstable, the results of FTF and FDA are almost stable.

\section{Experiments}

A schematic diagram of Experimental setup is shown in Fig. 10. The Experimental conditions in this paper are as follows.

(1) Athlon 1.4GHz CPU and RTLinux is used.

(2) Sampling frequency is $4,000 \mathrm{~Hz}$.

( 3 ) Filter lengths of FIR filters are 512.

(4) The bit length of AD converter is 12 bits, and DA converter is 16 bits.

( 5 ) The cut-off frequency of the low-pass filter used before AD conversion and after DA

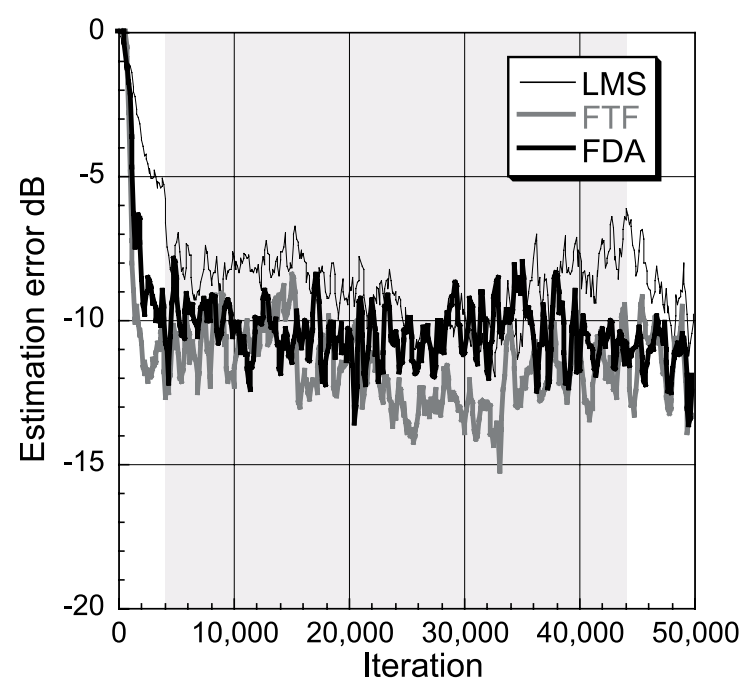

Fig. 8 Simulation: moving noise source speed : $0.10 \mathrm{~m} / \mathrm{s}$

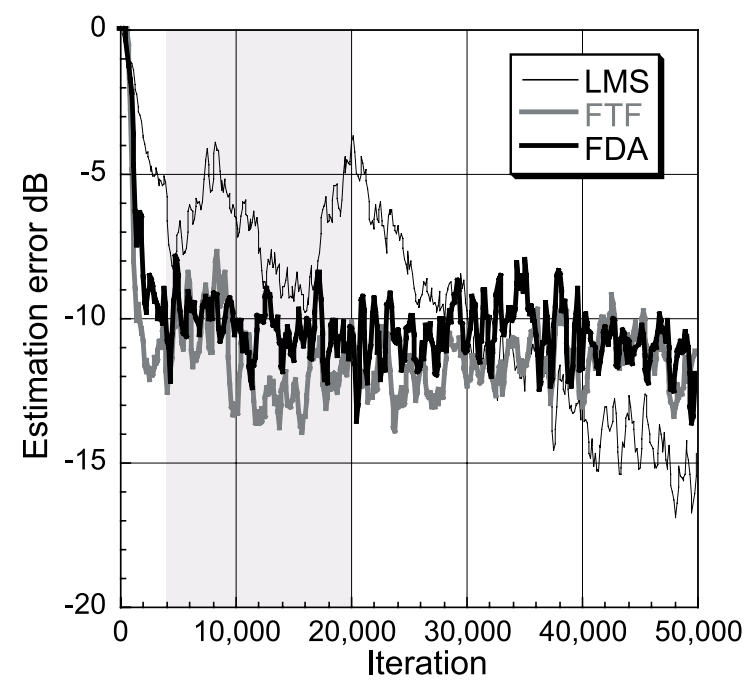

Fig. 9 Simulation: moving noise source speed : $0.25 \mathrm{~m} / \mathrm{s}$ 
conversion is $2,000 \mathrm{~Hz}$.

(6) The window length of FFT used in FDA algorithm is 512.

The signal $d$ in eq. (4) is estimated by

$$
\hat{d}_{k}=e_{k}-\widehat{\boldsymbol{g}} * \boldsymbol{y}_{k}
$$

where $\hat{d}_{k}$ is estimated value of the signal $d_{k}$, and $\boldsymbol{y}_{k}$ is the output signal.

\subsection{Fixed noise source}

Control results for a sound field caused by the fixed noise source is shown in Fig. 11. It can be confirmed that FTF and FDA converge at high speed same as the simulation results (Fig. 7). Because of the characteristics of the control sound source, the experimental result of FTF is worse than the simulation results.

\subsection{Moving noise source}

Control results for the moving noise source are shown in Figs. 12, 13, and 14. The speeds of the noise source are $0.1,0.25$, and $0.4 \mathrm{~m} / \mathrm{s}$ respectively in the experiments. In comparison with simulation results, particularly FDA algorithm is about to become slightly unstable. However, from these experimental results, it is shown that the control system keeps stability during control. We can see from experimental results, FTF algorithm shows better

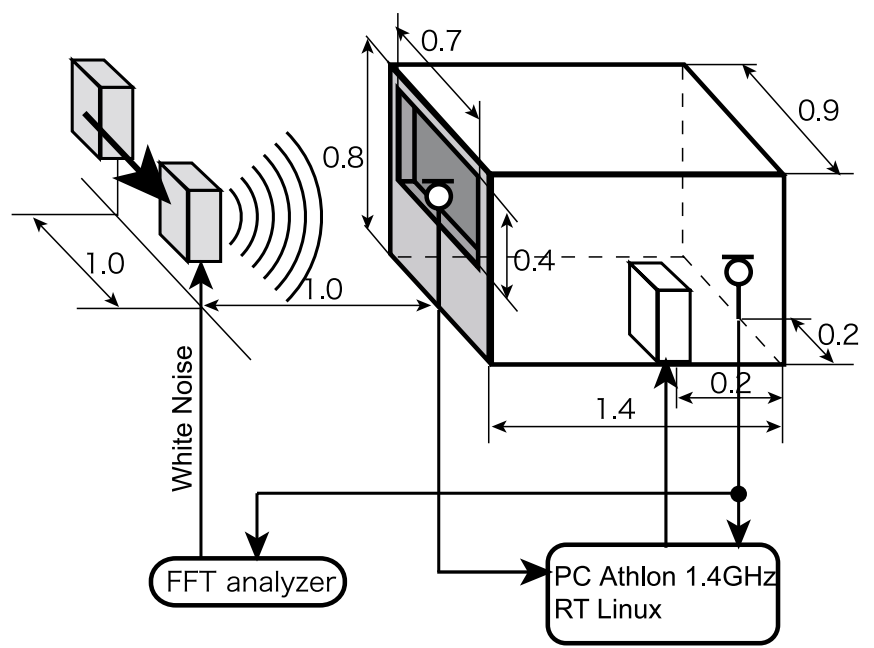

Fig. 10 Experimental setup

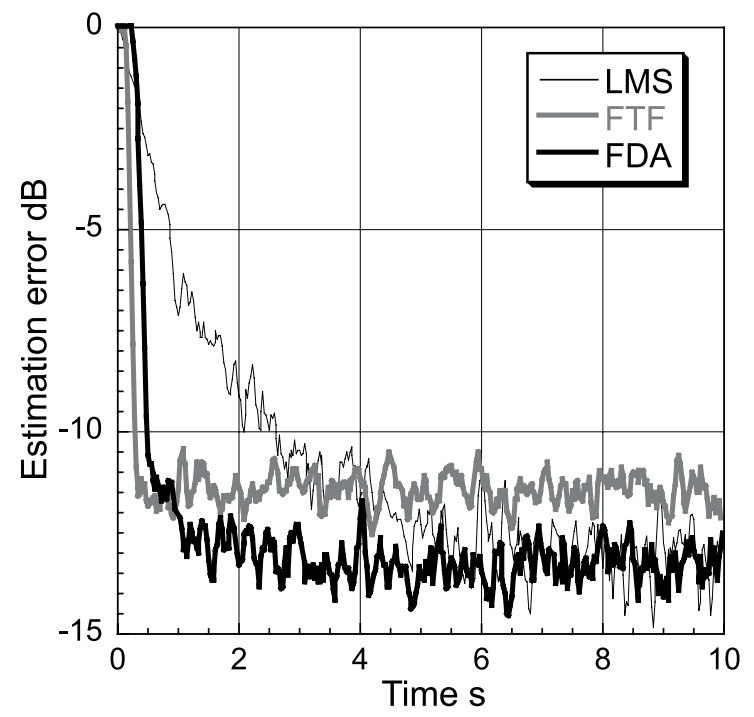

Fig. 11 Experiment: fixed noise source 
performance than both FDA and LMS do. It is also shown that improvements for FTF and FDA that proposed in this paper are effective.

\section{Conclusions}

In this paper, the effectiveness for the active noise control of both FTF and FDA algorithms that have high speed convergence characteristics compared with conventional LMS algorithm are confirmed in the three-dimensional sound field. Both FTF and FDA algorithms were improved to be able to be applied against the sound field caused by moving noise source. As a result, the following conclusions were obtained.

(1) In comparison with conventional LMS algorithm, both FTF and FDA converge at high speed against the fixed noise source.

(2) While the noise source is moving, FTF shows better experimental results than LMS does.

( 3 ) It is shown that FTF and FDA show better performance than LMS does under various conditions.

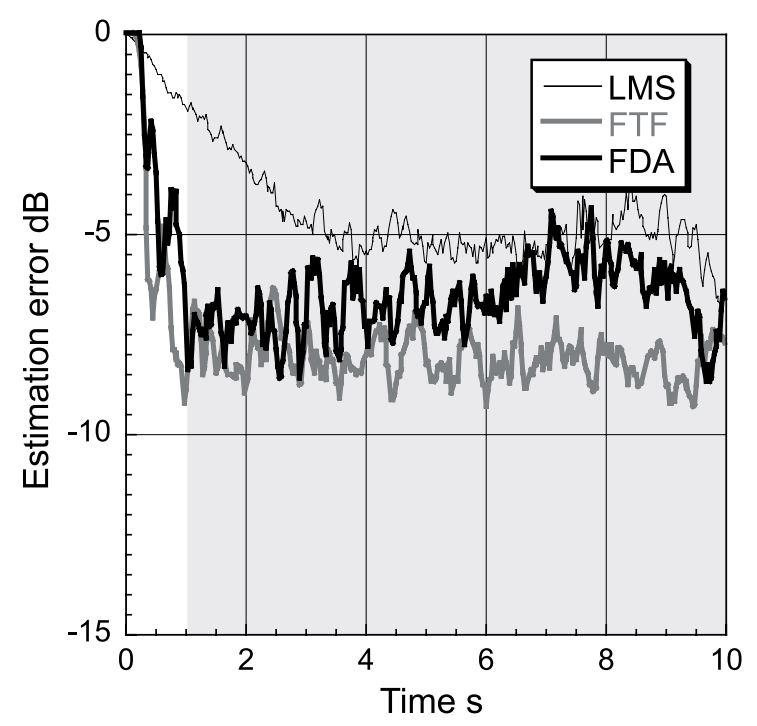

Fig. 12 Experiment: moving noise source speed : $0.10 \mathrm{~m} / \mathrm{s}$

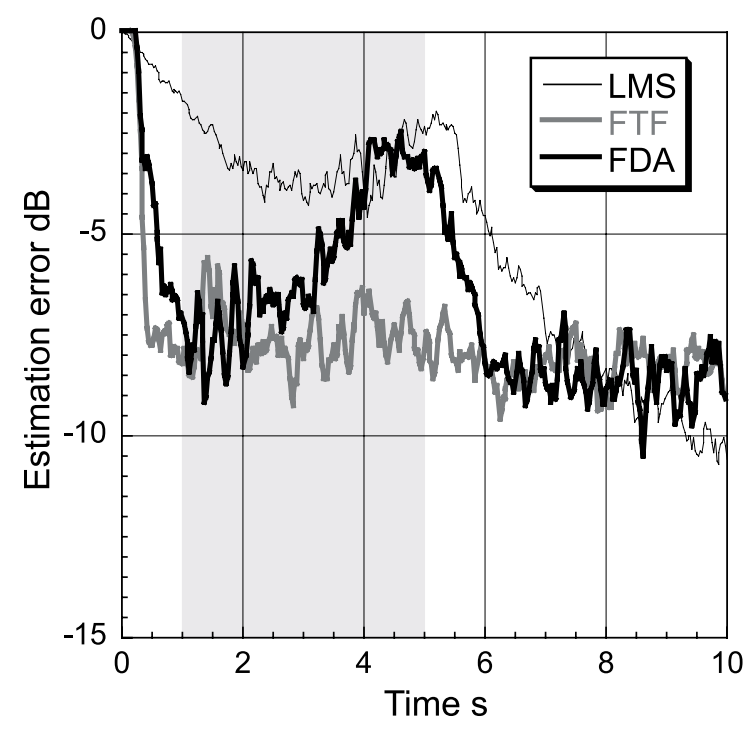

Fig. 13 Experiment: moving noise source speed : $0.25 \mathrm{~m} / \mathrm{s}$ 


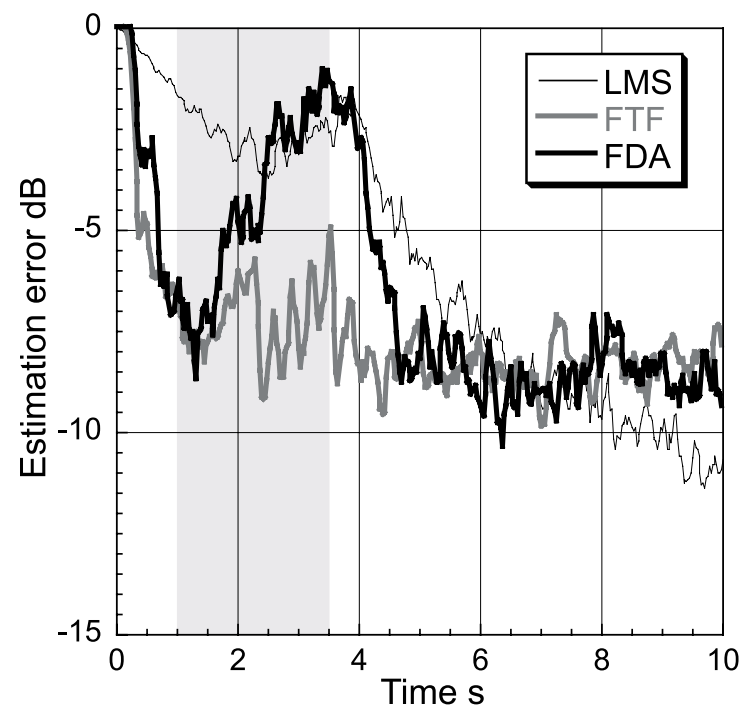

Fig. 14 Experiment: moving noise source speed : $0.40 \mathrm{~m} / \mathrm{s}$

\section{References}

( 1 ) S. Kijimoto, Y. Kanemitsu, K. Matsuda, D. Nakano, and M. Mifune, "Active Control of Sound Field Caused by Incoming Noise", Proc. of '99 Symposium on Environmental Engineering, 56-59, 1999

( 2 ) K. Yamamoto, Y. Kanemitsu, S. Kijimoto and K. Matsuda "Active Sound Control in a 3-Dimensional Sound Field caused by Incoming Noise”, Proc. of 2002 Symposium on Environmental Engineering, 127-129, 2002

( 3 ) B. Widrow and S. D. Stearns, Adaptive Signal Processing, Prentice-Hall, Englewood Cliffs, 1985.

( 4 ) S. Haykin, Adaptive Filter Theory, Prentice-Hall, 1996

( 5 ) Y. Iiguni, Adaptive Signal Processing Algorithms(in Japanese), BAIFUKAN CO., LTD, 2000

( 6 ) S. KIJIMOTO, Y. KANEMITSU, K. MATSUDA and H. SHIMOJIMA, "Frequency Domain Adaptive Algorithm for Active Noise Control", Proc. of Asia-Pacific Vibration Conference '97, I, 104-109, 1997

( 7 ) Y. Sasaki, Y. Kanemitsu, S. Kijimoto and K. Matsuda, "Active Noise Control in a 3Dimensional Sound Field wih FTF Algorithm”, Proc. of 2002 Symposium on Environmental Engineering, 130-133, 2002

( 8 ) A. NAGAMATSU, Introduction to Modal Analysis, CORONA PUBLISHING CO., LTD., 1993 